\title{
Quelques problèmes posés par la traduction en japonais de L'Occupation des sols
}

\section{Machiko Nakagawa}

\section{(2) OpenEdition \\ Journals}

Édition électronique

URL : http://journals.openedition.org/rsl/835

DOI : $10.4000 /$ rsl.835

ISSN : 2271-6246

Éditeur

Éditions Rue d'Ulm

Référence électronique

Machiko Nakagawa, "Quelques problèmes posés par la traduction en japonais de L'Occupation des sols ", Revue Sciences/Lettres [En ligne], $3 \mid$ 2015, mis en ligne le 08 décembre 2014, consulté le 19 avril 2019. URL : http://journals.openedition.org/rsl/835; DOI : 10.4000/rsl.835

Ce document a été généré automatiquement le 19 avril 2019

(c) Revue Sciences/Lettres 


\title{
Quelques problèmes posés par la traduction en japonais de L'Occupation des sols
}

\author{
Machiko Nakagawa
}

\section{Introduction}

1 Jean Echenoz affirme, à une table ronde qui réunit ses traducteurs de langues différentes, que la traduction fait disparaître inévitablement l'essentiel :

Je travaille sur un objet privilégié, la langue française. Mon objectif en dernière instance, après être passé par tout le travail de construction d'un récit, c'est le son. Le bruit que fait une phrase. Au service du son, j'essaie d'utiliser un ensemble d'outils poétiques: le rythme, les assonances, les allitérations, etc. J'essaie d'avancer sur une ligne de crête entre prose et poésie, ou plutôt dans une prose qui s'efforce d'annexer des outils poétiques permettant une distorsion de la prose, un écart, un jeu. Or tout ce travail sonore, qui me paraît toujours être l'essentiel, et qui mobilise pas mal d'énergie, de passion, il n'en reste rien quand on fait passer le texte dans une autre langue. Ce qui reste, c'est non pas le son, mais le sens, les images, la construction, qui en cours de route avaient fini par paraître secondaires ${ }^{1}$.

2 La sonorité du texte est au cœur du travail d'écriture de Jean Echenoz, de sorte que la traduction de ses textes dans une autre langue, transformant cet élément primordial, ne peut être fidèle. Lorsque l'on tente de traduire un texte en d'autres langues, on se trouve confronté à une impossibilité de cette nature. Notre travail part de ce point. Mais une transmission, la plus «fidèle» (Assouline, 2009) possible, est-elle tout de même envisageable? Un texte traduit peut-il produire le même effet sur les lecteurs non francophones dès la première lecture?

Notre problématique est donc de savoir comment transposer L'Occupation des sols, texte littéraire français, dans un autre système de signes (le japonais), sans réduire les spécificités linguistiques que recèle le texte original. Pour ce faire, il convient dans un 
premier temps de rappeler quelques caractéristiques du japonais, avant d'analyser le texte autour de la stratification des voix.

\section{Le japonais face au français}

4 Les catégories grammaticales observées dans une langue mettent au jour la présence ou l'absence des mêmes catégories dans une autre (Dhorne, 2005). Les caractéristiques du japonais se dégagent à la lumière du français, d'autant plus qu'il s'agit de langues différentes à tous les niveaux (phonétique, morphologique, lexical et syntaxique). Nous allons nous interroger sur les caractéristiques de la langue japonaise qui s'imposent lorsqu'on s'intéresse à la question de la stratification des voix dans les textes. Qu'est-ce qui fait entendre "plusieurs voix» dans L'Occupation des sols? Ducrot (1989) distingue trois instances narratives : le sujet parlant empirique (auteur), le locuteur (narrateur) et l'énonciateur (personnage). Dans le texte d'Echenoz il est très difficile de distinguer clairment entre le locuteur et les énonciateurs. "L'appareil formel de l'énonciation » proposé par Benveniste (1974) permet mieux de pister les traces de l'acte d'énonciation. Dans cette perspective, le japonais a une caractéristique qui s'affirme sous de nombreux aspects : l'importance du repère commun par rapport auquel se situent les interlocuteurs (Suzuki, Okonogi, Kitayama, 1981). Fréquentes sont les marques qui montrent que le locuteur prend en considération l'interlocuteur. Une importance particulière est ainsi accordée aux pronoms personnels et aux prédicats subjectifs. Il convient également de tenir compte du système d'écriture non seulement parce que nous traitons d'un écrit mais aussi parce que les formes de l'« hétérogénéité montrée » (Authier-Revuz, 1983) s'observent à travers les graphèmes.

5 Le couple « je »/ » tu » en japonais pose de nombreux problèmes. Tamba (1994) relève cinq caractéristiques des pronoms personnels en japonais en comparaison avec le français : 1 . le japonais n'a qu'une forme tonique $; 2$. les pronoms personnels japonais sont des morphèmes invariables; 3 . le verbe japonais ne varie pas selon le rang personnel et quand le sujet grammatical n'est pas désigné, l'énoncé est attaché, selon les cas, soit au locuteur, soit à l'allocutaire, à l'exception de l'usage du langage honorifique; 4 . le japonais a de multiples morphèmes pour un même rang personnel en fonction du sexe et du type de formulation; 5 . les relations interlocutives s'expriment en japonais au moyen $\mathrm{du}$ langage honorifique (moyens affixaux et lexicaux) alors que la prise en charge énonciative se marque par les particules finales ou par les adverbes modaux.

6 La notion de sujet grammatical elle-même, est, d'après Fujii (1991), importée de la grammaire européenne. Dans le souci de comprendre que la détermination de la valeur référentielle du locuteur se fait d'une manière différente en français et en japonais, deux points nous intéressent notamment: les multiples morphèmes de la personne et les relations interlocutives.

7 Le japonais offre divers termes pour exprimer l'identité des locuteurs. Kawaguchi (1984) affirme: «[...] il n'existe pas de forme "pronominale", neutre telle que l'on puisse l'employer sans considérations socio-culturelles». Par exemple, comme le note André Wlodarczyk, un homme qui parle le japonais peut se désigner, selon les circonstances, par des indicateurs de personne ou par un nom commun : BOKU (un indicateur de la première personne qui renvoie à une personne de sexe masculin, vis-à-vis de quelqu'un qui est égal du locuteur), PAPA (un père vis-à-vis de son enfant), SENSEI (un professeur vis-à-vis de son élève). En d'autres termes, il faut tenir compte des «problèmes du statut socio- 
culturel et de la reconnaissance du rôle que chaque personne joue dans l'acte de locution» (Wlodarczyk, 1996). Cette question est incluse dans celle du langage honorifique qui laisse des traces non seulement dans les prédicats verbaux mais également dans les noms, ce qui est d'autant plus important que l'adresse à l'allocutaire se fait très souvent sans le désigner directement. Ainsi, pour s'adresser à l'homme évoqué plus haut, on peut employer ANATA (un indicateur de la deuxième personne, de la part de quelqu'un qui est égal ou supérieur du locuteur/ de la part de son épouse), PAPA (de la part de son enfant ou de son épouse), SENSEI (de la part de son élève, de son collègue de travail).

8 En l'absence d'indicateurs explicites, certains types de prédicats renvoient aux instances énonciatives. Dhorne, Kawaguchi et Aoki (1995) relèvent trois prédicats impliquant, "sans marque explicite de détermination référentielle d'actants", la première (ou la deuxième) personne :1. les prédicats subjectifs ; 2 . la forme de suggestion du verbe ; 3 la forme impérative du verbe ${ }^{2}$. Par ailleurs, les particules finales ont pour fonction de marquer la modalité d'énonciation comme l'assertion, la confirmation ou l'interrogation..

Lorsque l'on compare le système du japonais à celui du français, les prédicats subjectifs constituent un point de différence remarquable. Au présent, certains adjectifs et certains verbes qui marquent la « subjectivité » s'emploient seulement à la première personne.

Sakana ga tabe tai.

(poisson + particule relationnelle + manger + suffixe verbal désidératif)

J'ai envie de manger du poisson.

10 Le suffixe indique que c'est la première personne qui est concernée, non la deuxième ni la troisième personne. Si l'on explicite le pronom personnel, seule la première personne est de ce fait convenable :

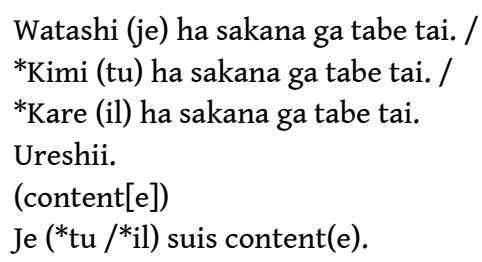

11 Cet énoncé n'est interprété qu'à la première personne. Les analyses de Dhorne, Kawaguchi et Aoki (1995) montrent toutefois que cette contrainte dépend du type d'énonciation ${ }^{3}$ :

En effet, le propre des prédicats subjectifs [...] c'est qu'ils impliquent en eux-mêmes une coalescence entre le sujet validant cet état et le sujet l'énonçant. Autrement dit, dans ce cas " je " n'est pas un autre. Ce n'est ni une description, ni un jugement mais un événement que le prédicat subjectif permet de construire en l'énonçant. (p. 240.)

12 À la deuxième et à la troisième personne, il faut changer la forme de ces verbes et adjectifs pour montrer un écart entre deux sujets, « le sujet validant cet état » et « le sujet l'énonçant ». Pour la deuxième personne, on ajoute un terme qui signifie la supposition darou : Kimi ha sakana ga tabe tai darou (Tu veux sans soute manger du poisson). Pour la troisième personne on ajoute un terme de conjecture : Kare ha ureshii ni chigainai (Il doit être content).

13 Dans la langue japonaise les prédicats subjectifs peuvent être employés par le locuteur premier (le narrateur) dans le récit à la deuxième et à la troisième personnes, comme si le point de vue du locuteur premier se superposait à celui du locuteur second, ce qui engendre un effet important dans la «narration ». 

texte littéraire, le locuteur premier (le narrateur) parle très souvent de façon «neutre ». Comme nous l'avons vu plus haut, un japonophone se repère à partir d'un critère qu'il partage avec l'interlocuteur. Le locuteur premier, en l'occurrence d'un texte littéraire, échappe à la catégorie porteuse de la situation socio-culturelle, parce qu'en général, l'on ne peut préciser le statut du locuteur ni celui de l'allocutaire. Supposons un texte écrit à la troisième personne : il présente la voix du locuteur, qui n'est pas identifiée socioculturellement. C'est là que l'on parle de « neutre ", notamment dans les écrits littéraires. Curieusement, cette voix «neutre » reflète la place qu'occupe la traduction elle-même dans la langue japonaise. Dans une perspective diachronique, la formation de cette instance se rapporte à l'introduction d'autres langues en japonais, qui suscite nécessairement l'opération de traduction. trois types de constituants dont les formes et les fonctionnements sont différents. Parmi eux, l'un est d'origine chinoise (KANJI) et les deux autres sont d'origine japonaise (KANA). Ce dernier se divise encore en Hira Gana et Kata Kana. Le KANJI, un idéogramme emprunté à la langue chinoise, sert à écrire les mots lexicaux tandis que le KANA, une écriture syllabique, sert à représenter les éléments grammaticaux comme les particules ou les terminaisons des verbes. Par ailleurs, les Kata Kana s'emploient exclusivement pour transcrire les termes étrangers et les onomatopées. On dispose de ces trois écritures. La disposition typographique présente une autre caractéristique: le japonais se lit verticalement et de droite à gauche, comme on le voit aujourd'hui surtout dans les œuvres littéraires ou dans les journaux ${ }^{4}$.

Cette graphie syncrétique illustre l'importance de la traduction en japonais. L'emploi des caractères chinois exige, dans un premier temps, une opération de traduction. En glosant les poèmes chinois de Soseki Natsume, un des écrivains modernes les plus célèbres dans l'histoire littéraire du Japon, Yoshikichi Furui, lui-même un des auteurs contemporains les plus importants, affirme que le japonais est « bilingue » du fait que chaque emploi du japonais passe par la traduction, la transformation de Hira Gana en KANJI (Furui, 2009). Fujii (1991) relève également qu'à l'époque de Meiji (1868-1912), l'écriture ne fut pas unifiée, disposant de cinq styles: 1. le style chinois (kanbun); 2. le style de traduction littérale des écrits chinois; 3 . le style japonais; 4 . le style épistolaire; 5 . le style de traduction littérale des écrits européens, que l'on choisit pour fonder «la langue nationale standard » (Fujii, 1991). Diachroniquement, la langue japonaise s'est formée à travers la lecture littérale des écrits chinois et la traduction littérale des écrits occidentaux (Yanabu, 1982; Maruyama, Kato, 1998). Ainsi, la voix « neutre » du locuteur (narrateur), essentielle pour la stratification des voix, s'est vue transposée dans le processus de l'unification de la langue écrite.

\section{Comment traduire la stratification des voix ?}

Notre objectif est de savoir comment transposer en japonais la superposition des voix que l'on observe dans L'Occupation des sols et comment créer chez le lecteur japonais les mêmes effets.

Cette problématique impose l'observation (ou l'interprétation) de la relation du locuteur premier aux énonciateurs. L'Occupation des sols étant écrit à la troisième personne, le

Revue Sciences/Lettres, 3 | 2015 
locuteur premier (le narrateur) raconte en japonais de manière « neutre ", dans la mesure où son statut socio-culturel n'est pas en question. Cependant, cette voix "neutre", adéquate pour le locuteur premier, se modifie à la rencontre des autres voix. Comme nous l'avons vu plus haut, le japonais n'explicite pas forcément le sujet grammatical. D'où le fait que «l'expression japonaise dépend de la situation de l'énonciation» et que «le locuteur ajoute souvent son point de vue aux référents de ses énoncés» (Sumi, 1987, p.111). Autrement dit, la traduction des voix entremêlées nécessite une prise en considération de la distance que prend le locuteur premier. Notre analyse vise d'abord à survoler dans l'ensemble la question du discours rapporté (3.1.) avant d'entrer dans une observation détaillée du premier paragraphe (3.2.) et du dernier paragraphe du texte (3.3.). Estelle Figon, professeure de japonais à l'École normale supérieure, a bien voulu retraduire en français notre traduction du premier et du dernier paragraphes de L'Occupation des sols, ce qui nous a permis de mieux cerner certains problèmes et de proposer une nouvelle traduction en japonais de certains passages.

\subsection{Remarques générales sur la traduction du discours rapporté}

Les énoncés brefs, attribués à Fabre au discours direct, ne pourraient pas être traduits en japonais sans tenir compte de la relation qu'il entretient avec son auditeur, en l'occurrence dans la plupart des cas, son fils Paul.

(1) Ça ne se rend pas, soupirait Fabre en posant une main sur sa tête, sur ses yeux, et le découragement l'endormait.

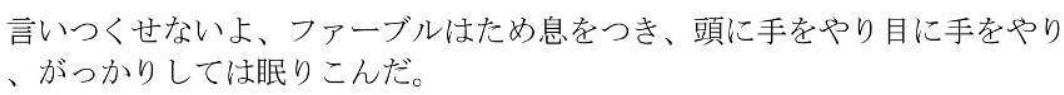

Iitsukuse nai yo, Faabru ha tameiki wo tsuki, Atama ni te wo yari me ni te wo yari, gakkarishite ha nemurikon da.

(2) Regarde un peu ta mère, s'énervait Fabre que ce spectacle mettait en larmes, en rut, selon.

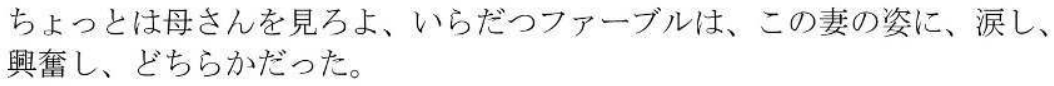

Chotto ha kaasan wo miro yo, iradatsu Faabruu ha, kono tsuma no sugata ni, namidashi, koufunshi, dochiraka datta.

L'énoncé "Ça ne se rend pas » dans (1) pourrait être traduit en "Iitsukuse nai yo »: le verbe Iitsukusu (tout dire) + le verbe modal négatif nai + la particule finale " yo». Kawaguchi (1984) explique que :

Les grammairiens décrivent cette PF [particule finale] comme «emphatique». En fait, cette PF sert à mettre l'accent sur la dissymétrie entre locuteur et interlocuteur en conférant à celui-ci une position " passive »; mais en même temps, dans le cadre du dialogue qui est une condition nécessaire de son emploi, elle met face-à-face ces protagonistes. (p. 123)

Dans (2), l'impératif « Regarde un peu ta mère » se traduirait en japonais de même par la particule finale «yo» et par la forme impérative du verbe «miru (regarder)» (la forme employée exclusivement par les hommes) : «Chotto ha kaasan wo miro yo » («Un peu » + «Maman » + particule accusative + « regarde » + particule finale). Ici se pose la question de l'appellation de la mère. Si l'on traduit «ta mère " mot à mot, il faudrait « kimi no hahaoya » («tu/toi »+ «de »+ « mère »). Notre choix « Kaasan» est un terme par lequel 
l'énonciateur appelle la mère. Fabre se met à la place de son fils Paul et utilise « Kaasan " pour désigner Sylvie : « ta mère ».

En accentuant l'expression du découragement ou de l'irritation et en prenant en compte la présence de l'auditeur Paul, les énoncés en question se dégagent dès lors de la voix du locuteur premier (narrateur). Pourtant, sans crochets (presque équivalents en japonais aux guillemets ${ }^{5}$ ), ni particule citative «to», marqueurs du discours rapporté, le texte traduit ne met pas en relief les énoncés de Fabre sur le plan de la disposition graphique.

Par rapport aux énoncés de Fabre, « un peu plus disert que Paul », la voix du fils s'entend moins directement. C'est là que l'on retrouve la question de la distance du locuteur premier à l'énonciateur.

(3) Paul considérant cela, une femme qui venait sur le trottoir s'arrêta derrière lui, leva les yeux au ciel et cria Fabre. Paul, dont c'est quand même le nom, se tourna vers celle qui criait Fabre Fabre encore, j'ai du lait. La voix énervante tomba du ciel, d'une haute fenêtre au milieu du ciel : tu simules, Jacqueline. La femme s'éloignait, on ne sait pas qui c'était. Monte, Paul.

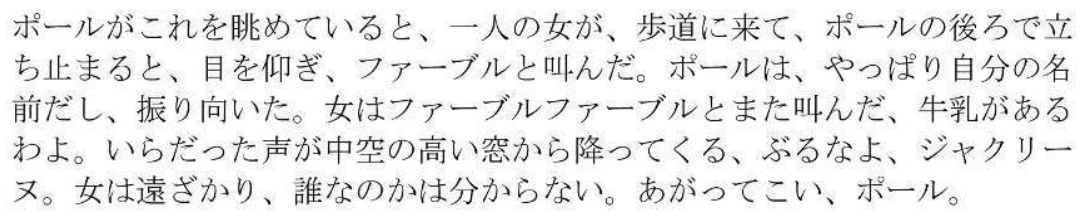

Pooru ga kore wo nagame te iru to, hitori no onnna ga, hodou ni kite, Pooru no ushiro de tachidomaru to, me wo aogi, Faabru to sakenda. Pooru ha, yappari zibun no namae dashi, furimuita. Onna ha Faabru Faabru to mata sakenda, gyunyu ga aru wa yo. Iradatta koe ga chukuu no takai mado kara futte kuru, buru na yo, Jakuriinu. Onna ha toozakari, dare na no ka ha wakara nai. Agatte koi Pooru.

24 Ici nous observons les formes marquées du discours de Fabre et de la femme («Fabre», « Fabre Fabre », « tu simules, Jacqueline », « Monte, Paul »). La voix de Paul apparaît ici et là sans « émettre » un mot. Dans l'énoncé «Paul, dont c'est quand même le nom », on traduit le pronom relatif «dont» et l'article défini «le», qui n'existent pas en langue japonaise, par le terme «zibun » qui désigne la première personne : «Pooru ha, yappari zibun no namae dashi, » (Paul + particule thème + quand même + je/moi + de + nom + verbe modal affirmatif + particule conjonction). Nous avons essayé ainsi de faire résonner à l'intérieur du discours du locuteur, la voix de la première personne, Paul.

L'énoncé «on ne sait pas qui c'était » pose le problème du pronom «on». En japonais, traduit par l'absence du sujet grammatical, il pourrait être compris comme le locuteur premier, comme l'énonciateur Paul ou bien l'interlocuteur, à savoir le lecteur. La voix de Paul intervient ainsi dans celle du locuteur premier.

(4) C'était un sépulcre au lieu d'une effigie de Sylvie, on l'approchait d'un autre pas, d'une démarche moins souple.

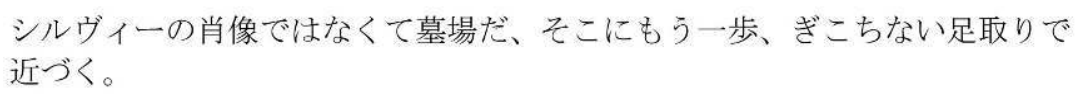

Sirubii no shouzou de ha naku te hakaba da, sokoni mou ippo, gikochinai ashidori de chikazuku.

On peut lire dans "C'était un sépulcre au lieu d'une effigie de Sylvie» la présence de la voix intérieure de Paul. L'absence de conjonction et de point final à la suite de cet énoncé accélère le rythme en liant les deux propositions, si bien que le sujet « on » contient aussi 
le locuteur premier, Paul et le lecteur. En japonais, "Sirubii no shouzou de ha naku te, hakaba da, sokoni mou ippo, gikochinai ashidori de chikazuku.» (Sylvie + de + effigie + verbe modal + particule thème + négation + particule conjonction + sépulcre + copule, làbas + un autre pas + maladroit + marche + particule manière + approche), la différence majeure réside dans la syntaxe qui exige qu' " au lieu d'une effigie " précède "c'était un sépulcre». Par conséquent, le discours intérieur de Paul s'entend dans «c'était un sépulcre ». En effet, l'énoncé doit commencer par " au lieu d'une effigie de Sylvie», une description objective, et passer ensuite à "c'était un sépulcre", une observation subjective. Cette tension grandit dans l'énoncé suivant, "on l'approchait», où nous n'avons pas traduit le sujet grammatical « on », ce qui fait que le point de vue peut être appréhendé de nouveau comme celui du locuteur premier, de Paul et du lecteur.

La stratification des voix du locuteur premier et de Paul crée parfois une distance à l'égard du discours de Fabre.

(5) C'était une belle robe au décolleté profond, c'était une mère vraiment.

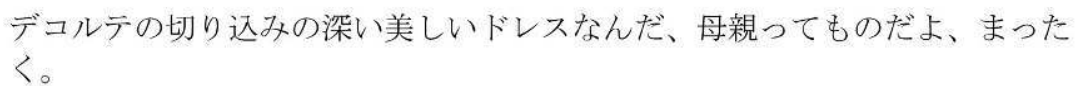

Dekorute no kirikomi no fukai utsukushii doresu nan da, hahaoya tte mono da yo, mattaku.

Dans « c'était une mère vraiment ", on perçoit l'ironie. D'après la théorie polyphonique de Ducrot (1989) «parler de façon ironique, cela revient, pour un locuteur L, à présenter l'énonciation comme exprimant la position d'un énonciateur $\mathrm{E}$, position dont on sait par ailleurs que le locuteur $\mathrm{L}$ ne prend pas la responsabilité et, bien plus, qu'il la tient pour absurde» (p. 211). Dans (5) se superposent les voix du locuteur premier et de l'énonciateur Paul. L'énonciation mise à distance est dès lors celle de Fabre, « Regarde un peu ta mère ». Comment transposer de la même manière cette polyphonie dans la traduction? Nous avons employé en partie la langue parlée: "hahaoya tte mono da yo, mattaku.» (mère + particule apposition + chose + copule + particule finale + vraiment ). La particule «tte » est une forme familière de «to iu»; la particule finale «yo» n'est pas employée, comme nous l'avons vu plus haut, dans la narration censée être « neutre».

\subsection{Le premier paragraphe}

\section{Texte de L'Occupation des sols}

[E1]Comme tout avait brûlé [E2]- la mère, les meubles et les photographies de la mère -, [E3]pour Fabre et le fils Paul c'était tout de suite beaucoup d'ouvrage: [E4]toute cette cendre et ce deuil, déménager, courir se refaire dans les grandes surfaces. [E5]Fabre trouva trop vite quelque chose de moins vaste, [E6]deux pièces aux fonctions permutables sous une cheminée de brique dont l'ombre donnait l'heure, et qui avaient ceci de bien d'être assez proches du quai de Valmy.

Notre traduction :

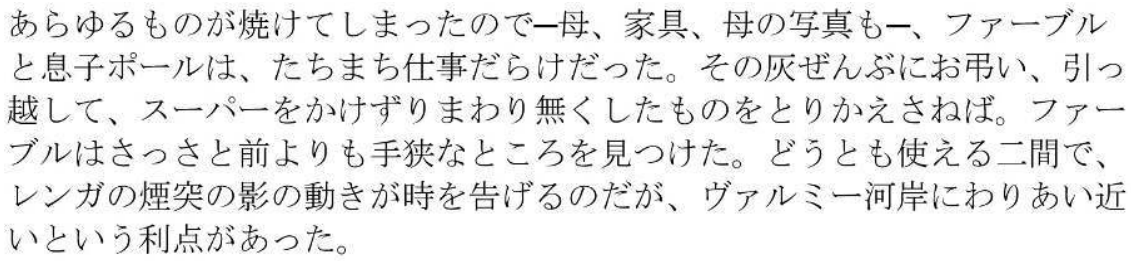

Retraduction en français par Estelle Figon : 
Comme tout avait brûlé - la mère, les meubles et les photos de la mère - Fabre et son fils Paul, furent soudain submergés de travail. En plus de toutes ces cendres et des obsèques, il avait fallu déménager et courir les supermarchés pour tout remplacer. Fabre trouva très vite un nouveau lieu encore plus exigu que le premier: deux pièces interchangeables où l'ombre de la cheminée de briques donnait l'heure et qui avaient pour avantage une relative proximité avec le quai de Valmy.

\section{Commentaires}

[E1] « Comme tout avait brûlé »

Arayuru mono ga yakete shimatta node

tout + chose + particule nominatif + brûlé + avait +comme

- « tout " : en japonais, il y a un certain nombre d'options pour « tout ». En effet, notre premier choix était «Minna », un substantif pronominal employé autant pour les choses que pour les personnes. Mais l'emploi de « tout » exclut les personnes. Aussi serait-il plus approprié d'utiliser en japonais le substantif « mono (chose) ».

[E2] « - la mère, les meubles et les photographies de la mère -, »

haha (la mère), kagu (les meubles), haha (la mère) no (de) shashin (les photographies) mo (aussi) -,

- «la mère" : Le mot japonais " haha » est un nom commun. Mais ce mot s'emploie aussi comme " ma mère ", en présence de l'autre, et en même temps par une femme pour référer à elle-même à l'intention de son enfant. Cette ambiguïté pourrait perturber la lecture et amener le lecteur à se poser une question : qui parle?

[E3] « pour Fabre et le fils Paul c'était tout de suite beaucoup d'ouvrage : »

Faaburu to musuko Pooru ha tachimachi shigoto darake datta.

Fabre + et + fils + Paul + particule thème + tout de suite + ouvrage+ beaucoup + était.

- «c'était tout de suite beaucoup d'ouvrage ": «ce» est impossible à transposer, si bien que l'on est obligé de modifier la syntaxe. La retraduction révèle des défauts de notre traduction provenant de l'effacement de l'ambiguïté référentielle de «ce». Par ailleurs, notre proposition ne garde pas le sens de l'expression « c'est...beaucoup de ».

Deuxième proposition de traduction :

$$
\text { ファーブルと息子ポールには、直ちに大しごとだった。 }
$$

Faaburu to musuko Pooru ni ha, tadachini ooshigoto datta.

Fabre + et + fils + Paul + pour, + tout de suite + un ouvrage massif + était

[E4] « toute cette cendre et ce deuil, déménager, courir se refaire dans les grandes surfaces.»

Sono hai zenbu ni otomurai, hikkoshishi, Suupaa wo kakezurimawari, nakushita mono wo torikaesa neba.

cette + cendre + toute + et + [ce] deuil, + déménager, + les grandes surfaces + particule accusatif + courir, + perdu + chose + particule accusatif + regagner $+[$ il $]$ faut.

- « toute cette cendre ${ }^{6} »$ : nous avons deux possibilités pour traduire « cette » : « kono» et « sono ». En général, le démonstratif « ko » délimite « l'aire auto-centrée du locuteur » et «so » «l'aire opposée de l'interlocuteur ». Dans ce contexte, l'emploi de » kono » instaure d'emblée les énonciateurs (Fabre et Paul) ; si l'on utilise « sono ", cela revient à montrer une distance que prend le locuteur premier par rapport aux énonciateurs, tout en se mettant dans une même zone intradiscursive. D'un autre côté, l'usage de "so" est à même de garder les valeurs aussi bien démonstrative qu'anaphorique. D'ailleurs, il apparaît que dans la première traduction, on ne peut distinguer « cette cendre » de "ces cendres ». Puisque la pluralité ne s'exprime pas en japonais dans le seul substantif, il serait certes difficile de 
transposer « cette cendre ». Pour ce faire, on peut ajouter néanmoins le substantif «yama» (« montagne » en français) qui signifie aussi l'état où s'entassent les choses ou les personnes.

- « ce deuil » : le démonstratif « ce » nous semblait à première vue se traduire, par le préfixe honorifique «o », qui indique que le locuteur premier instaure une certaine relation avec les locuteurs seconds ou bien qu'il s'agit des voix des personnages. Mais le résultat de la retraduction (« les obsèques ») révèle un écart entre le texte original et la traduction. Le mot équivalent à « deuil » en japonais est «mo » qui signifie plutôt le processus nécessaire pour les proches de la personne décédée mais qui ne contient pas le sens de «douleur». Nous choisissons dès lors d'ajouter le terme « kurushimi » (« douleur »).

- « déménager, courir [...]» : pour traduire à la lettre l'infinitif, on pourrait mettre " koto (fait)» à la fin du verbe, en montrant que le verbe fonctionne comme un nom. Choisir le terme "neba», qui signifie l'obligation, revient à le concevoir comme l'impératif. Cette interprétation nous a conduit à négliger l'ambiguïté sur laquelle jouent ces énoncés. Ceux-ci peuvent être en effet interprétés comme une énumération dans la liste ou un simple constat. Soucieux d'intégrer dans la traduction cet effet, nous avons opté pour la forme conclusive pour chaque verbe.

Deuxième proposition de traduction :

$$
\begin{aligned}
& \text { この灰のやまとこの喪の苦しみ、ひっこしする、スーパーをかけずり生活を } \\
& \text { たてなおす。 }
\end{aligned}
$$

Kono hai no yama to kono mo no kurushimi, hikkoshisuru, suupa wo kakezuri seikatsu wo tatenaosu.

cette + cendre + particule possessif + montagne + et + ce + deuil + particule possessif + douleur, + déménager + la grande surface + particule accusatif + courir + la vie + reconstruire

\subsection{Le dernier paragraphe}

\section{Texte de L'Occupation des sols}

[E1]Le soleil en effet balaierait tout le studio, comme un projecteur de poursuite dans un music-hall frontalier. [E2]C'était dimanche, dehors les rumeurs étouffées protestaient à peine, parvenant presque à ce qu'on les regrettât. [E3]Ainsi que tous les jours chômés, les heures des repas tendraient à se glisser les unes sur les autres, [E4]on s'entendit pour quatorze heures - ensuite on s'y met. [E5]Un soleil comme celui-ci, développa le père de Paul, donne véritablement envie de foutre le camp. [E6]Ils s'exprimèrent également peu sur la difficulté de leur tâche qui requerrait, c'est vrai, de la patience et du muscle, puis des scrupules d'égyptologue en dernier lieu. [E7]Fabre avait détaillé toutes les étapes du processus dans une annexe agrafée aux plans. [E8]Ils mangèrent donc vers quatorze heures mais sans grand appétit, leurs mâchoires broyaient la durée, la mastication n'était qu'horlogère. [E9]D'un tel compte à rebours on peut, avant terme, convoquer à son gré le zéro. [E10]Alors autant s'y mettre, autant gratter tout de suite, [E11]pas besoin de se changer, on a revêtu dès le matin ces larges tenues blanches pailletées de vieille peinture, [E12]on gratte et des stratus de plâtre se suspendent au soleil, piquetant les fronts, les cafés oubliés. [E13]On gratte, on gratte et puis très vite on respire mal, on sue, il commence à faire terriblement chaud. 
太陽は実際、国境付近にあるミュージックホールのピンスポットライトのご とく、部屋中にふりそそぎそうだった。日曜なのだが、外は、不服そうにボ ソボソとざわつき、うっとうしくなりかけていた。失業中の日々のように、 食事の時間はどんどんずれこみがちで、二人は十四時ということで了解した 一そして開始する。こんな太陽は、ポールの父を明るみにさらし、本当にも うずらかりたい。父子はまたこの任務の難しさについても、ほとんど語らな かった。二人の任務は、確かに、忍耐と筋力、そして最後のところでは、エ ジプト考古学者の細心さを要するものとなろう。ファーブルは、図面にホッ チキス留めした添付文書に、全工程を細かく書き出していた。父子はちやん と十四時に食べたが、あまり食欲もなく、あごは時間をすりつぶし、咀嚼は 時を刻むのみ。こんなカウントダウンなら、終わりが来る前に、好き勝手に ゼロにできる。さあもう始めた方がいい、すぐにけずる方がいい、着替える 必要もないし、古いペンキがあちこちに飛んだ白い大きな作業着を朝から着 ている、削る、すると石膏の層雲が太陽にひっかかり、額につきささり、コ

41 Retraduction en français par Estelle Figon

Le soleil, en réalité, comme les projecteurs de poursuite des music-hall frontaliers, semblait vouloir couler à flots dans la chambre. C'était dimanche, au dehors on percevait une agitation sourde et comme mécontente, une sorte de morosité s'installait. Comme les jours de chômage, l'horaire des repas avait tendance à se relâcher et ils s'entendirent sur deux heures - ensuite ils commencent. Le soleil éclaire vivement le père de Paul, on a envie de ficher le camp. Le père et le fils n'ont presque rien dit au sujet de la difficulté de cette tâche. Celle-ci nécessiterait sans doute persévérance, force physique, et à la fin, une minutie d'égyptologue. Fabre, sur un document qu'il a agrafé sur le plan, avait noté précisément toutes les étapes des travaux. Le père et le fils mangèrent bien à quatorze heures, mais ils n'avaient pas beaucoup d'appétit, leurs mâchoires broyaient le temps et seule leur mastication découpait les heures. Avec un tel compte à rebours, avant que la fin n'arrive,( ?) Allez, mieux vaut commencer maintenant, mieux vaut gratter tout de suite, on n'a pas besoin de se changer, on a enfilé dès le matin les amples combinaisons blanches constellées de vieilles taches de peinture, on gratte, et à mesure les nébuleuses de plâtre s'accrochent au soleil, picotent les fronts, on a oublié le café. Gratter, gratter, puis tout de suite on a du mal à respirer, on sue, il commence à faire terriblement chaud.

\section{Commentaires}

[E2]C'était dimanche, dehors les rumeurs étouffées protestaient à peine, parvenant presque à ce qu'on les regrettât.

Nichiyo nanoda ga, soto ha fufuku so ni bosoboso to zawatsuki, uttoshiku narikake teita.

dimanche + est + mais, dehors + particule thème + d'une manière mécontente + onomatopée + particule citatif + faire du bruit, irritant + devenir presque + avait.

43 - La difficulté principale consiste à traduire les deux verbes « protester » et " regretter». Notre proposition a tenté, en analysant les sèmes de chaque terme, de le remplacer par d'autres éléments : «les rumeurs» par le verbe, «étouffées » par l'onomatopée qui représente la manière dont on parle à voix basse ; "protester » par les adverbiaux. En regard de la retraduction « C'était dimanche, au dehors on percevait une agitation sourde et comme mécontente, une sorte de morosité s'installait ", on aperçoit néanmoins une déviation considérable de la traduction. Nous cherchons ici à saisir " protester » à la lettre :

日曜のことで、外ではざわめきが押さえつけられて、ちよっとだけ不平を訴 え、なんだか物足りない。 
Nichiyo no koto de, soto deha zwameki ga osaetuske rarete, wazukani fuhei wo uttae, nandaka monotarinai.

dimanche + c'est, + au dehors + rumeurs + [particule nominatif] + sont étouffées, + à peine + protester,+ légèrement + peu suffisant.

[E5]Un soleil comme celui-ci, développa le père de Paul, donne véritablement envie de foutre le camp.

Konna taiyo ha, Pooru no chichi wo akarumi sarashi, hontoni mou zurakaritai.

tel + soleil + [particule thème], Paul + de + père + [particule accusatif] + en lumière + exposer, vraiment + déjà + avoir envie de foutre le camp. due à la méprise du sens de « développer ».

$$
\text { こんな日差しでは、とポールの父はとく、本当にもうずらかりたい。 }
$$

konna hizashi de ha, to Pooru no chichi ha gochiru, hontouni mou zurakaritai.

tel + soleil + particule causal + particule thème, + particule citatif + Paul + particule possessif + père + développer + vraiment + déjà + avoir envie de foutre le camp.

[E9]D'un tel compte à rebours on peut, avant terme, convoquer à son gré le zéro.

Konna kaunto daun nara, owari ga kuru maeni, sukikatteni zero ni dekiru.

tel + compte à rebours + si, + fin + [particule nominatif] + venir + avant + à son gré + pouvoir faire le zéro.

- « [...] convoquer à son gré le zéro »: Puisque le texte retraduit montre bien la faute de notre traduction, nous proposons :

\section{好き勝手にゼロをよびだせる。}

Sukikatteni zero wo yobidaseru.

à son gré + zéro + [particule nominatif] + pouvoir convoquer.

[E10]Alors autant s'y mettre, autant gratter tout de suite, [E11]pas besoin de se changer, on a revêtu dès le matin ces larges tenues blanches pailletées de vieille peinture, [E12]on gratte et des stratus de plâtre se suspendent au soleil, piquetant les fronts, les cafés oubliés. [E13]On gratte, on gratte et puis très vite on respire mal, on sue, il commence à faire terriblement chaud.

- " piquetant les fronts » : Il s'est révélé à partir de la retraduction que l'interprétation du mot au sens de « piquer » n'est pas correcte. Nous proposons :

$$
\text { 額にてんてんと飛び }
$$

Hitai ni tentento tobi

front + à + piqueter

- « on a revêtu [...]»: Le japonais n'exige pas le sujet «on» dans ce passage. Cette omission fait entendre dans E11 le locuteur second qui parle, dont nous ne savons plus si c'est le père ou le fils.

- « on gratte, on gratte [...]»: En traduisant ce dernier énoncé, nous pourrions lire les prédicats subjectifs dans « on respire mal » et « il commence à faire terriblement chaud». Effectivement, les adjectifs « ikigurushii » et «atsui » que nous avons employés sont des prédicats de sensation, réservés à la première personne. L'omission du sujet implique du reste la présence de la première personne. En contrepoint, se discerne la voix qui garde une distance par rapport à cette première personne. En temps normal, une succession de verbes appelle des terminaisons différentes. Ici, toutefois, nous avons préféré appliquer la forme conclusive à chacun des verbes, comme si chacun terminait la phrase. La juxtaposition des verbes en cette forme constitue, en l'absence des particules finales, une série de phrases affirmatives "neutres ». Mais on ne peut discerner quelle voix vient de qui.

Revue Sciences/Lettres, 3 | 2015 


\section{Conclusion} formes corrélées en japonais et à explorer toutes les possibilités qu'offre cette langue. En comparaison avec le texte d'Echenoz, notre traduction en japonais demeure pourtant hétérogène.

\section{constat:}

D'un autre côté, il est réconfortant de penser que malgré cette déperdition des livres puissent exister dans une autre langue, et dans ces conditions c'est peut-être l'étranger plus que la France qui m'autorise à me définir comme romancier ${ }^{7}$.

Si l'on trouve, au sein de l'hétérogénéité matérielle, « une identité à partir d'une altérité » (Blanchot, 1971, p. 72.), ce sera à travers la lecture, dont le centre de gravité, auquel renvoient sans cesse les voix entremêlées, demeure la perte irréparable de la mère.

\section{BIBLIOGRAPHIE}

« Echenoz et ses traducteurs », Translittérature, n 16, 1998.

Assouline, Pierre, « Traduction littéraire et trahison littéraire », hebdomadaire du Monde, $\mathrm{n}^{\circ} 7$, 2009.

Authier-Revuz, Jacqueline, « Hétérogénéité(s) énonciative(s) », Langages, 73, 1983, p. 98-111.

Benveniste, Émile, Problème de linguistique générale, t. II, Paris, Gallimard, 1974.

Blanchot, Maurice, L'Amitié, Paris, Gallimard, 1971.

Dhorne, France, Kawaguchi, Junji, Aoki, Saburô, « La personne en japonais », Langues et langage. Mélanges offerts à A.Culioli, Paris, PUF, 1995, p. 237-246.

Dhorne, France, « Où le comportement exceptionnel ne justifie pas l'exception », D'une langue à l'autre. L'acte du colloque Besançon 5-6-7 septembre 2002, Presses universitaire de Franche-Comté, 2005, p. 45-50.

Ducrot, Oswald, Le Dire et le dit, Paris, Éditions de Minuit, 1989.

Fujii, Noriko, Historical discourse analysis : grammatical subject in Japanese, Berlin, New York, Mouton de Gruyter, 1991.

Furui, Yoshikichi, Soseki no kanshi wo yomu (Lectures des poèmes chinois de Soseki), Tokyo, Iwanamishoten, 2009.

Hayashi, Midori, Interlocution et polyphonie : une relecture du discours indirect libre : une étude contrastive portant sur le français et le japonais, thèse de doctorat sous la direction d'Oswald Ducrot, Paris, EHESS, 1998. 
Kawaguchi, Junji, « Le concept de personne », Emile Benveniste aujourd'hui. Actes du Colloque international du CNRS Université François Rabelais Tours, 28-30 septembre 1983, t. I, Paris, Peeters, 1984, p. 119-124.

Maruyama, Masao, Kato, Shuichi, Honyaku to nihon no kindai (La traduction et la modernité japonaise), Tokyo, Iwanamishoten, 1998.

Meschonnic, Henri, Pour la poétique II. Épistémologie de l'écriture. Poétique de la traduction, Paris, Gallimard, 1973.

Sumi, Yoichi, Honyaku futsu bunpo (La grammaire française pour la traduction), t. II, Tokyo, Nihon Honyakuka Yosei Center, 1987.

Suzuki, Takao, Okonogi, Keigo, Kitayama, Osamu, « Nihonjin no kokoro to kotoba (L'esprit et la langue des Japonais) », Eureka, n 13-2, 1981.

Tamba, Irène, « Démonstratifs et personnels en japonais. Deixis et double structuration de l'espace discursif », La deixis. Colloque en Sorbonne (8-9 juin 1990), Paris, PUF, 1992, p. 187-195.

Wlodarczyk, André, Politesse et personne. Le japonais face aux langues occidentales, Paris, L'Harmattan, 1996.

Yanabu, Akira, 1982, Honyakugo seiritsu jijo (Mots inventés à travers la traduction), Tokyo, Iwanamishoten, 1996.

\section{NOTES}

1. «Echenoz et ses traducteurs », p. 16.

2. En ce qui concerne la conjugaison, les verbes en japonais subissent une altération en fonction des suffixes ou des termes qui les suivent. A titre d'exemple, pour le verbe « hashiru (courir) », la voyelle varie comme suit: hashir-a-nai (ne pas courir), hashir-o-u (courons!), hashir-i-tai (avoir envie de courir), hashir-u (courir), hashir-e-ba (si l'on court), hashir-e (cours !). Ce qu'on appelle ici « la forme de suggestion » et « la forme impérative " renvoient respectivement à la deuxième ( hashir-o-u/ courons!) et la sixième (hashir-e / cours!), sur lesquelles on ne s'attarde pas davantage ici. La quatrième (hashi-ru) forme se nomme "conclusive", comme nous le verrons plus tard.

3. Par exemple, pour l'énoncé «Ureshii ? ", l'intonation montante qui signifie l'interrogation fait comprendre qu'il s'agit de l'interlocuteur, la deuxième personne. L'autre exemple que citent Dhorne, Kawaguchi et Aoki est un énoncé dans la publicité, "Tsumetai bîru ga nomi tai, sorenara Suntory " (Vous avez envie de boire une bière fraîche, alors Suntory) qui est traduit en français nécessairement à la deuxième personne mais qui est attaché à «toute personne susceptible de valider ce désir ». (Dhorne, Kawaguchi, Aoki, 1995)

4. Il n'empêche qu'aujourd'hui beaucoup de textes adoptent la lecture horizontale à l'occidentale.

5. Les crochets ne sont pas indispensables en japonais au discours direct.

6. Par ailleurs, il est probable que le terme « cendre » évoque chez un lecteur japonais les restes mortels parce que l'on effectue traditionnellement l'incinération.

7. « Echenoz et ses traducteurs », p. 16. 


\section{RÉSUMÉS}

L'Occupation des sols de Jean Echenoz - récit marqué par une polyphonie curieuse - est difficile à traduire dans une autre langue. Comment une traduction peut-elle être "fidèle" au texte original, en transposant la stratification des voix? La présente étude examine, autour de cette spécificité linguistique, comment traduire notre corpus en japonais, en considérant une caractéristique japonaise de la narration. À partir de là, notre propos se focalise sur le discours rapporté afin de recréer les mêmes effets dans la version traduite. Du reste, une retraduction par Mme Estelle Figon en français nous permet de tester notre traduction tout en mettant en lumière les problèmes posés. Toutefois, l'impossibilité d'une équivalence absolue entre le texte original et sa traduction ne les empêche pas de montrer tous deux où se trouve le centre de gravité, à savoir, la perte irréparable de la mère d'un personnage.

Jean Echenoz's Plan of Occupancy-a narrative characterised by a curious polyvocality-is difficult to translate in another language. How can a translation be 'faithful' to the original, while rendering the stratification of voices? From this viewpoint, this study explores the problems of translating this text in Japanese-specifically by considering a Japanese characteristic of the narration and by focussing on speech representation to recreate the same effects in the translated text. In addition, a retranslation of the first and last paragraphs, by Prof. Ms. Estelle Figon, into French enables us to test our translation as well as to outline its problems. However, the impossibility of complete equivalence between the original and the translation does not necessarily prevent them from each showing where lies the centre of gravity, that is, an irreparable loss of a character's mother.

\section{INDEX}

Mots-clés : Jean Echenoz, traduction, linguistique, voix, japonais

Keywords : translation, linguistics, voice, Japanese

\section{AUTEUR}

\section{MACHIKO NAKAGAWA}

Chargée de cours, à l'Université Keio (Tokyo, Japon).

Parmi les publications :

«"Les besognes des mots" chez Georges Bataille : l'exemple du Petit », Cahiers d'études françaises, 14, 2009, p. 48-63.

«La fonction stratégique de l'instant dans "Postulat initial" de Georges Bataille », Cahiers d'études françaises, 17, 2012, p. 34-49.

«La Maison brûlée ou une tentative cinématographique de Georges Bataille », Geibun Kenkyu, 104, 2013, p. 196-219. 